dass zur Zeit seiner Entstehung dort ein wärmeres tropisches Klima geherrscht hat $(7,8,10)$.

Die Übereinstimmung der Entstehung von Bauxit und Laterit wird jedoch von manchen bestritten und für den Bauxit eine Zufuhr von tonerdehaltigen Lösungen, vielleicht im Zusammenhang mit postvulkanischen Vorgängen angenommen $(6,16,18,24,28)$.

Fassen wir zum Schluss das Ergebnis der bisherigen Untersuchungen noch einmal kurz zusammen. so können wir sagen: Laterit besteht im wesentlichen aus Tonerdehydraten, namentlich aus Hydrargillit, meist gemischt mit Eisenoxyd oder Brauneisenerz. Seine Entstehung ist darauf zurückzuführen, dass in den Tropen wegen der geringen Menge von Humusstoffen im Boden die hydrolytische Wirkung des Wassers auf die Silikate rein zur Geltung kommt, während die Verwitterung im gemässigten Klima in erster Linie unter Mitwirkung von Kohlensäure erfolgt.

B. Unter der Redaktion der deutschen geologischen Gesellschaft.

\title{
Ergebnisse neuerer Spezialforschungen in den deutschen
} Alpen.

\section{Allgämer dlpen und angrenzende Gebiete.}

\author{
Von F. Felix Hahn (München).
}

\section{Literatur.}

1. Ampferer, O., Bemerkungen zu den von A. Heim und A. Torrquist entworfenen Erklärungen der Flysch- und. Molassebildung am nördlichen Alpensaum. Verh. geol. Reichsanst. Wien 1908. p. 189 and Entgegnung an Tonnquist. Verh. geol. Reichsanst. Wien 1909. p. 43.

2. - u. Otnesonge, Th., Uber exotische Gerölle in der Gosau und verwandte Ablagerungen der tirolischen Nordalpen. Jahrb. geol. Reichsanst. Wien 1909. 59. p. 289.

3. - Uber den geologischen Bau der Berge des Sulzeltales. Jahresber. 16. Akad. Alpenklub, Innsbruck 1909.

4. Aus den Allgäuer und Lechtaler Alpen. Verh. geol. Reichsanst. Wien 1910. p. 58 .

5. Freduer, 0., Uber Versteinerungen aus den Arlbergschichten bei Bludenz und einige neue Fundorte von Flyseh and Aptychenkalken im oberen grossen Walsertal Voraribergs. Zeitschr. d. geol. Ges. 56. 1904. Briefl. Mit. p. 8 .

6. HAAs, A., Zum geologischen Bau der Umgebung des Formarin-Sees in den Lechtaler Alpen. (Mit Kartenskizze 1:70000.) Mitt. Geol. Ges. Wien 1909 II. p. 384 .

7. Haniel, C. A., Vorläufige Mitteilung über das Vorkommen von Gosaukreide südlich des Hohen Lichts. Verh. seol. Reichsanst. Wien 1908. N. 16. - Die geologischen Verhältnisse der Südabdachung des Allgaùer Hauptkammes und seiner südlichen Seitenäste vom Rauhgern bis zum Wilden. Mit Karten skizze. 1 : 75000. Zeitschr. d. geol. Ges. 63. 1911. 
8. Mruıus, H., Die geologischen Verhältnisse des hinteren Bregenzer Waldes in den Quellgebieten der Breitach und der Bregenzer Ach bis südlich zum Lech. Mit einer Karte 1:25000. Landeskundl. Forsch. geogr. Ges. München 1909. Heft 5.

9. Pontoppidan, H., Die geologischen Verhältnisse des Rappenalpentales sowie der Bergkette zwischen Breitach und Stillach. Mit einer Karte 1:25000. Geogn. Jahreshefte München 24. 1911.

10. Röscr, A., Der Kontakt zwischen dem Flysch und der Molasse im Algäu. Mit einer Karte 1:25 000. Mitt. geogr. Ges. München I. Heft 3. 1905.

11. Rollier, Id, Die Entstehung der Molasse anf der Nordseite der AJpen. Vierteljahrsschr. naturf. Ges. Zürich 1904.

12. Rothpletz, A., Das Gebiet der zwei grossen rhätischen Überschiebungen zwischen Bodensee und dem Engadin. Sammlung geol. Führer $(\mathrm{X})$ durch die Alpen I. (Bornträger). Berlin 1902.

13. - Geologische Alpenforschungen II. Ausdehnung und Herkunft der rhätischen Schubmasse. München 1905.

14. Schulze, G., Die geologischen Verhältnisse des Allgäuer Hauptkammes von der Rotgundspitz bis zum Kreuzeck und der nördlich ausstrahlenden Seitenäste. Mit einer Karte 1:25000. Geogn. Jhrh. München 18. 1905.

15. Tonnquist, A., Vorläufige Mitteilung über die Allgäu-Vorarlberger Flyschzone. Sitzber. K. preuss. Ak. Wiss. 30. 1907. p. 591. - Die Allgäu-Voralberger Flyschzone und ihre Beziehung zu' den ostalpinen Deckenschüben. Mit einer Karte 1:75000. N. Jhrb. f. Min. 1908 I p. 63.

16. - Noch einmal die Allgän-Vorarlberger Flyschzone und der submarine Einschub ihrer Klippenzone. Verh. geol. Reichsanst. Wien 1909. p. 37.

17. W FPfer, E., Die nördliche Flyschzone im Bregenzer Wald. Mit einer Karte $1: 25000$. N. Jhrb. f. Min. Beilagebd. 27. 1908.

Wenn auch im Gegensatz zu den alpinen Arbeiten des vorigen Jahrhunderts, die vorwiegend von stratigraphischen Gesichtspunkten geleitet waren, heute das tektonische Rätselbild viel mehr zu fesseln scheint, so haben doch die letzthin unternommenen Detailaufnahmen auch eine Menge für die Kenntnis der Schichtfolge bedeutsamen Materials beigebracht, das zunächst zusammengestellt werden soll.

a) Stratigraphische Resultate.

Innerhalb der ostalpinen Deckenmasse (im Sinne von STEINMANN $^{1}$ ) und SuEss ${ }^{2}$, hier $=$ rhätische Schubmasse von Rothpcerz $[12,13]$ ) hat sich für die Trias die von E. Böse ${ }^{3}$ ) vorgenommene Aufstellung eines eigenen Bündner Faziesbezirks wenigstens in seiner Unterscheidung von einem östlichen bayerischen Bezirk bewährt, wenn auch wohl jene Bezeichnung kaum hierfür beizubehalten ist.

1) Geolog. Beobachtungen in den Alpen I. Ber. naturw. Ges. 9, 10. $1895 / 99$. Freiburg a. Geolog. Probleme des Alpengebirgs. Zeitschr. deutsch-öst. Alp.Ver. 37. 1906.

:) Antlitz der Erde III. 1910.

3) Beitr. zur Kenntris der alp. Trias. Zeitschr. d. geol. Ges. 50. 1898. 
Die tiefere Trias bayerischer. Entwickelung ist nur in Schubfetzen von Bundsandstein and Muschelkalk am Rande der Lechtaler Überschiebung und an der Basis von Innenschuppen der Hindelanger Gegend bekannt geworden (13), während der Wettersteinkalk an drei Stellen (bei Reutte und Weissenbach am Lech, mit dem Klotz der Heiterwand, bei Imst) sich gegen Westen vorschiebt.

Von der Vorarlberger Faziesreihe sind wenig abweichender Muschelkalk (,Virgloria Kalk") und Arlbergschichten mit regem Gesteinswechsel (300-500 m mächtig) im oberen Lechtal.(8) wie südlich des Formarin Sees (6) beschrieben worden; letztere haben auch bei Bludenz (5) gute Fossilien geliefert, wodurch ihre ladinische Stellung gessichert erscheint. Die Raibler sind in Gestalt geringmächtiger, fossilleerer Gipse und Rauhwacken oft schwer von der Unterlage zu trennen, besitzen jedoch weitere Verbreitung, wie ursprünglich angenommen wurde $(7,8,14)$.

Vom eintönigen, fossilarmen Hauptdolomit (500-1000 m) lässt sich nur unscharf etwa $50 \mathrm{~m}$ mächtiger Plattenkalk abscheiden, der nirgends die Bedeutung erlangt, die ihm gewöhnlich in dem bayerischen Faziesbezirk zukommt.

Die Mächtigkeit des $\mathrm{Rh} \ddot{\mathrm{a}} \mathrm{t} \mathrm{s}$ schwankt in weiten Grenzen zwischen 25 und $200 \mathrm{~m}$, je nachdem auch Merge? oder Kalke die Oberhand gewinnen; in der Fauna fällt ein merkliches Zurücktreten der tieferen schwäbischen Lamellibranchiaten-Fazies gegenüber der Brachiopodenreichen Kössener und karpatischen Entwickelung auf. Die hangende Gesteinspartie neigt zur Bildung oberrhätischer Korallen- und Megalodonten Kalke, ein zeitlich, doch nicht faziell übereinstimmendes Äquivalent der oberrhätischen Riffkalke der östlicheren Nordalpen und früher unter dem Sammelnamen der Dachsteinkalke einbezogen, deren Art der Entstehung sie ja vollkommen teilen.

Wichtiges und zum Teil überraschender Natur sind die Funde in den jüngeren Schichtserien, deren obere Hälfte den älteren Autoren fast ganz entgangen war.

Zunächst ist eine grosse Gesetzmässigkeit der liassischen Sedimentation ${ }^{1}$ ) unverkenmbar: $\alpha$ bis $\gamma$ wird gewöhnlich durch rote und graue 3-10 $\mathrm{m}$ umfassende Zephalopodenkalke mediterranen Charakters vertreten, die nur selten Verwandtschaff zu Hierlatz, häufiger zu Adneter-Fazies aufweisen. Sie werden von fleckenerfüllten Mergeln und Kalken bedeckt, die in ihrer Fauna (spärlich Formen von $\beta$, meist nur von $\gamma$ bis $\zeta$ ) viel mehr mitteleuropäische Züge erkennen lassen; von $\delta$ an sind nur mehr vereinzelte mediterrane Elemente $\mathrm{zu}$ finden.

Dieser 300 bis $500 \mathrm{~m}$ starke Verband von "Allgäus chichten" birgt nun nicht nur fast den gesamten Lias (obere Stufe durch

1) F. Frux Hahn. Neue Funde in nordalpinem Lias der Achsenseegegend und bei Ehrwald. N. J. f. Min. Beilage Bd. Heft 3. II. 1911. 
manganreiche Schiefer ausgezeichnet), ein nicht unbeträchtlicher oberer Teil muss als Doggeräquivalent betrachtet werden. So scheidet Haniel (7) eine hierher gehörige, bis $15 \mathrm{~m}$ dicke Reihe dunkler Kieselkalke, dünner Mergellagen und kristalliner Krinoideenkalke aus und AMPFERER ${ }^{1}$ ) (2) konnte von den Vilseralpen her gegen Südwest vorschreitend oolithische, kieselige, krinoideenreiche Kalkbänke zwischen Fleckenmergel und Radiolarite eingeschaltet und zum Teil mit letzteren wechsellagernd nachweisen. (Profil vom Kirchendach nahe des Vilsalpsees.)

Die höheren Juraglieder (meist etwa $250 \mathrm{~m}$ stark, stellenweise bis $\mathrm{zu} 500 \mathrm{~m}$ anschwellend) lassen sich in roten und grünlichen Radiolarit ( $30 \mathrm{~m}$; höherer Dogger? und MaIm) und hangende bald grace, bald rot und grünlichgraue Aptychenkalke und Nergel (Tithon; mit Spongiennadeln, Radiolarien und Calpionella a lpina Lor.!) zerlegen.

Das stärkste Interesse beanspruchen die so lang verkannten kretazeischen Ablagerungen. Konkordant auf die Aptychenschichten legen sich, allerdings nur an einzelnen geschützten Stellen erhalten, ne okom e Mergel, durch Zephalopodenfunde AMPFERER's (4) sichergestellt; auch den „Kreideflysch" Haxiel's (7) von Holzgau möchte ich hier umbedenklich einreihen.

Dass diese älteren Kreideablagerungen so überaus spürlich verbreitet sind, darf sicher in erster Linie der Abrasionswirkung des transgredierenden Meeres der Oberkreide zugerechnet werden $(2-5,7-9,13,14)$. Mit oft recht grobklotzigem Grundkonglomerat (Kalkalpine Gerölle und solche der Grauwackenzone, keine hochkristallinen Geschiebe) beginnend und im eigentlichen Allgäu fast stets auf Fleckenmergel and oberen Jura, in den Lechtaler Alpen auf Hauptdolomit abgelagert, erreichen diese buntgemischten Schichten (Brekzien, Konglomerate, Sandsteine und teilweise foraminiferenreiche (7) Mergel in letzterem Gebiet die grössten Höhen (bis $2800 \mathrm{~m}$ ) und sehr bedeutende Mächtigkeiten. Während die schönen Funde Haniel's (7, Hippuriten, Gastropoden, Lamellibranchiaten in guter Übereinstimmung mit den Pattenauer und Gerhardsreuter Mergeln der belvetischen Vorzone Oberbayerns) das senone Alter eines grossen Teils dieser Ablagerungen gewährleisten (Gos a u im allgemeinen), ist ein anderer dem $\mathrm{Zenoman}$ zuzuweisen, dass mit seiner leitenden Orbitulina concava schon bis Gramais (4) verfolgt wurde $\left.{ }^{2}\right)$.

Jünger als obersenone Sedimente sind dagegen bis jetzt in diesem Bezirk der ostalpinen Decke noch nicht bekannt geworden.

1) Vergl. auch Jahresber. geol. Reichsanstalt. Wien 1908. p. 15.

2) Die bisher angewandte Bezeichnung "Flysch“ für derlei transgressive Kreide der ostalpinen Decke ist demnach trotz gelegentlicher Gesteinsähnlichkeit am besten wohl fallen zu lassen. 
Die helvetisch-lepontinische Vorzone umfasst eine oberjurassische bis mitteltertiäre Schichtenreihe. Über den älteren Teil derselben liegen leider neuere Arbeiten noch nicht vor, doch dürften in nächster Zeit, von der MrLus'schen Aufnahme der Gegend von $\mathrm{Au}$ und Bezau auch in stratigraphischer Hinsicht (tiefhinabreichender Hochgebirgskalk des oberen Jura w. a. m.) interessante Beiträge zu erwarten sein.

Zwischen Schrattenkalk und Gault liegt nach WePFer (17). ein deutlich erkennbarer Erosionshiatus; der Gault $(10-15 \mathrm{~m})$ ist ziemlich fossilreich, petrographisch schon merklich vom linksrheinischen verschieden und geht nach oben kontinuierlich in Seewer$\mathrm{kalk}$ (Zenoman) und foraminifrenreiche graue oder rötliche Seew ermergel (Turon, Senon), beide oft schwer trennbar und auch zum Teil in Wechsellagerung (zusammen etwa $250-500 \mathrm{~m}$ ), über. Recht beachtenswert ist einmal eine Partie dunkler, sandiger Kalkmergelschiefer der Gegend von Andelsbuch, die teils in reinen dunklen Kalk, teils in echte helle Seewer übergehen und nach guten Funden WepFER's eine wohl ins mittlere Senon gehörige Fauna von Gastropoden und Lamellibranchiaten bergen, dabei wiederum unverkennbare Beziehungen zu Pattenauer and Gerhardtsreuter Mergel des östlichen Oberbayerns bekunden. In anderer Weise zeigen die dickbankigen

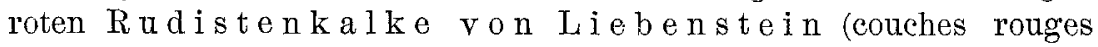
nach SteInmans) Verwandtschaft mit ostalpiner Kreideausbildung; sie sind aber von hellen foraminiferenreichen Seewermergeln begleitet und nur als eigenartige Differenzierung der Seewerkalke aufzufassen. Die obersen onen Schichten des Grünten and der Oberstdorfer Gegend sind leider noch immer nicht gründlich bearbeitet worden, so dass vorerst das ReIs'sche ${ }^{1}$ ) Schema: untersenone Seewer - Burgberggrünsandstein (eine nach OSO auskeilende fazielle Vertretung der tieferen Nierentaler) - schwärzliche, zum Teil sandige Mergel and Kalke (= Pattenauer und Gerhardtsreuter Mergel) - grobkörnige glaukonitische Sandsteine des Burgbühls bei Oberstdorf (= Hachauer; oberstes Senon) zu gelten hat.

Ưber die obereSeewerkreide, nach übereinstimmenden Beobachtungen nirgends tiefergreifend, legt sich der Flysch, nach ToRNQUisT (15) mit einem groben Basalkonglomerat beginnend, das keinesfalls tektonischen Ursprungs ist. Leider haben bis jetzt die meisten der vorliegenden Spezialaufnahmen der Eigengliederung des Flyschs, der unbedingt noch durchgeführt werden muss, zu wenig Beachtung geschenkt, nur ToRNQuist (Versuch kartographisch ausgeschiedener Zonentrennung:) und WEPFER verdanken wir wichtige Angaben. Nach ihnen lässt sich ein liegender chon-

1) Geol. Karte der Vorderalpenzone zwischen Bergen und Teisendorf. Geogn. Jahresh. VIII 1894/95. p. 17 u. ff. 
dritenreicher Mergelkalkverband, dann sandige Schichten mit dunklen Schieferlagen (etwa $250 \mathrm{~m}$ ), eine zweite kalkig-mergelige Zone (ungefähr $90 \mathrm{~m}$ ) und $100 \mathrm{~m}$ mächtige, dickbankige Sandsteine im Hangenden unterscheiden; etwa in der Mitte des ganzen Komplexes soll nenerdings eine Konglomeratlage zu finden sein und auch die kristallinen Gerölle und Blockmassen wären in die eigentliche Flyschsedimentation einzubeziehen. Recht bedeutungsvoll ist endlich die Angabe ToRnquist's, dass im lieg enden Teil hausgrosse Linsen von $\mathrm{Nummulitenkalk}$ dem Flysch normal eingelagert sind. Feinkörnige kieselige Lagen (Kieselschiefer, Toneisensteine und Kalkhornsteine) werden als halbmetamorphe Bildungen (Verkieselung und tektonischer Druck) abgesondert.

Die an den Flysch angrenzende Molasse (nur sie soll hier besprochen werden) ist nach den übereinstimmenden Beobachtungen WEPFER's und Rösch's (10 besimmbare Pflanzenfunde!) hauptsächlich als oberoligozän (Aquitanien) zu betrachten, im Gegensatz zur Ansicht RoLlier's (11), der miozänes Alter (Nagelfluhbänke!) annahm. WEPFER fand $\mathrm{zu}$ unterst hellgraue, glimmerige, feste Sandsteine mit Kohlespuren und Fliessformen, darauf kommen graue sandige Mergel mit Cyrena subarata Schloth. (2 $\mathrm{m})$, einige Meter Sandsteine und die erste, etwa $40 \mathrm{~m}$ dicke Nagelfluhbank, dann wieder Sandsteine mit Pechkohlenflözchen von $250 \mathrm{~m}$ Mächtigkeit, eine zweite Nagelfluhbank (wieder etwa $40 \mathrm{~m}$ ) und darüber bunte liolasse (oberstes Oligozän).

Von glazialen Ablagerungen findet man überall Moränen kalks alpiner Vereisung, zentralalpine Zuflüsse sind dagegen für das Illergebiet kaum anzunehmen, da die spärlich anzutreffenden kristallinen Geschiebe aus den Konglomeraten der Oberkreide und des Flysches abgeleitet werden können; allerdings hat mehrfach ein kräftiges Überquellen des Lechgletschers $(8,9)$ stattgefunden. Über die zwischen Moränen eingeschalteten kohligen Lagen von Sonthofen (nach PENCK ${ }^{1}$ ) entweder interglazial oder interstadial, Achsenschwankung?) liegen keine neueren entscheidenden Unterswchungen vor, dagegen haben Tornquist und WepFer eine Reihe von Talstufenterrassen nachgewiesen und auch kartographisch festgelegt.

\section{b) Ausbau destektonischen Bildes.}

Die Besprechung des Gebirgsbaus unseres Alpenteils wird sich naturgemäss an die grossen tektonischen Einheiten halten müssen, deren Klarlegung wir A. Rothpletz (13), G. Steinmans ${ }^{2}$ ) und F. E. Suess verdanken. Wir kommen so von Nord nach süd vorschreitend aus der Zonesubalpiner Molas e in das helvetisch-lep ontinische Gebiet der Schweizerkreide und des Flysches und steigen

1) Alpen im Eiszeitalter I. 1902, p. 256.

2) Siehe Anmerkung 1) u. ') p. 208. 
sodann über den weithin erkennbaren Rand der ostalpinen Decke (= rhätische Schubmasse Rothpletz non Steinmand ${ }^{1}$ ) empor. Für den südlichen Teil der Molas senzone kommt Wepfer zu dem Schlusse, dass in ihm die Molasse mit einer Synkline beginnt, die nur strekenweise überstürzt, an der Bregenzer-Ach dagegen normal liegt; gegen den Flysehkontakt stellen sich die ältesten Schichten ein. Die meist steil Süd fallende, doch auch gelegentlich ebenso nordgeneigte (10) Trennungsfläche zur helvetischen Zone besitzt einen schnurgerade verlaufenden, nur ganz selten etwas gestaffelten Ausstrich; an ihr brechen sich die kräftigen Querstörungen der Flyschzone (15). Die Anpressung des helvetischen Gebiets kann jedoch bis zu steiler Überschiebung gehen (so östlich Dornbirn), ja die eigenartigen ortsfremden Schöllchen von Kreide und Eozän westlich Sonthofen, die ein beträchtliches Stück ins Molassegebiet vordringen (10), machen es recht wahrscheinlich, dass ehemals flache Schubkontakte vielleicht viel mehr verbreitet waren, als sie es heute sind; eine sekundäre Versteilung der Trennungsfläche beider Zonen als Ausfluss des Rücksinkens der tieferen alpinen Massen unter der wuchtigen ostalpinen Deckenlast erscheint immer mehr im Bereich der Möglichkeit.

Die Kreide-Flyschzone des Bregenzer Waldes ist leider noch keineswegs genügend tektonisch bekannt geworden, als dass sich heute etwa schon eine Zerteilung in verschiedene Deckenelemente rechtfertigen liesse. Verhältnismässig gut begangen ist nur der nördliche Flyschzug westlich der Iller (1, 13, 15-17), doch gerade er birgt tektonische Spezialfragen schwierigster Art. Kräftige Reduktion der eigentlichen Flyschverbreitung und dafür eintretende Bereicherung der Schichtenfolge hat hier auch feinere Schollengliederung gezeitigt. Eine energische Verfaltung von Seewerkreide und vorwiegend eozänem Flysch, Einschaltung von Bruchstücken fremder Decken und deutliche Durchfurchung mit Quersprüngen of t bedeutender Verschubweite charakterisiert nun das tektonische Bild.

WEPFER hat es dann zwar, vornehmlich auf Grund scheinbar anormalen Kontaktes von Flysch zu Kreide versucht, eine Gliederung: in liegende Kreide-, mittlere Flysch- und hangende Kreidedecke für die Gegend zwischen Dornbirn und Bregenzer-Ach vorzuschlagen, allein die herbe Kritik AMPFERER's ${ }^{2}$ ) hat wohl mit Recht die allzugeringe Unterlage für solche Zerspaltung ans Licht gezogen; immerhin könnte der kräftige nördliche Vorstuss der Kreide bei Andelsbuch, der hier eine völlige Unterbrechung des Flyschzugs herbeiführt (die Flysch-Kreidegrenze ToRNQuist's und WePFER's jedoch inhomogen!) auf flachere Deckbewegungen zurückzuführen sein.

1) Da die Bezeichnung „rhätiseh" in ganz verschiedenem Sinne in der alpinen Literatur angewandt wird, sei hier "ostalpine" trotz der Prioritat des ROTHPT.ETz'schen Namens angenommen.

2) Verh. geol. Reichsanst. Wien 1909. p. 206. 
TORNQQUST's Untersuchungen galten vor allem den eigenartigen Juraklippen des Feuerstätter Kopfes und den kristallinen Blockresten im Flysch. Bezüglich ersterer wird tektonische Zugehörigkeit zur ostalpinen Schubmasse, deren Faziesbereich das Gestein auch entstammt, submariner Aufschub und sekundäre Versteilung der Kontaktfläche verbunden mit randlicher Verfaltung von Klippe und Flysch recht wahrscheinlich gemacht; auch AMPFERER's Einwürfe (1, Ableitung der Klippe vom Untergrund) haben den überzeugenden Argumenten ToRNQUIST's einigermassen weichen müssen, wenn ersterer auch wohl mitErfolg einen Zusammenhang mit dem Vordringen der Lechtaler Schuppe abweist. Die kristallinen Reste, deren Auffassung als tektonische Mitbringsel der Deckenverschübe heute fast überall beigepflichtet wird, hält ToRNQUist für normal in den Flysch einsedimentiert, eine Ansicht, die durch gelegentliche Verfaltung eines Granitblock führenden Flyschverbands in die Kalkklippe wohl wesentlich gestützt wird.

Die südlich angrenzende helvetische Kreide ist durch eine mehrfach kräftig gestaffelte tektonische Fläche begrenzt, die von Dornbirn bis ins Illertal den Charakter einer bald steil Süd oder ebenso Nord einschliessenden „Längsverwerfung“ (ROTHPLETZ ${ }^{1}$ ) 13) beibehält; für eine flache Auflagerung der Kreide sind bis jetzt westlich der Iller sichere Anzeichen nicht bekannt geworden (vielleicht bei Andelsbuch?; Gegeneinanderstreichen von Flysch und Kreide in Linie Besler-Bolgen, 15), aber die Verhältnisse östlich dieses Flusses (völliges Fehlen der pördlichen Flyschzone am Grünten) mahnt auch hier zu Vorsicht vor allzu rascher Entscheidung.

Von der helvetischen Kreidezone selbst ist bis heute wenig Neues zu berichten, hier moderne tektonische Gesichtspunkte zur Geltung: zu bringen ist zunächst der Mruius'sehen Aufnahme der Gegend von Au und Bezau vorbehalten; sie wird sich auch mit der Ansicht E. Blumer's (Säntisgebirge, Bern 1905) auseinandersetzen müssen, der ja die Fortsetzung seiner Hochkastendecke in der Kreide des Bregenzer Waldes zu finden glaubte. Hier möge nur auf bemerkenswerte Anzeichen verschieden gerichteter Druckkräfte hingewiesen werden, die EckenT aus der Struktur des Gottesackerplateaus am Hohen Ifen ablas (Hauptspaltenrichtung in OSO-WNW, verschiedene kreuzende Nebenspalten), und für die auch WEPFER in der Verbiegung seines Weissenfluhsattels neue Beweise brachte.

Fast noch dürftiger sind neue Forschungsresultate in der süd lichen Flyschzone von Feldkirch bis Hindelang trotz der Spezialaufnahmen zwischen Bregenzer-Ach und Oberstdorf, die sich leider mit der Feststellung nach Nord überkippter, eng gepresster Falten begnügen. Und doch muss jetzt schon eine Erscheinung als recht auffällig bezeichnet werden. PontoppIDan (9) hat $5 \mathrm{~km}$ südlich der helvetischen Kreide mit Flysch verquälte Seewermergel vom Fidere-

1) Auch Geol. Alpenforschungen I. München 1900. 
pass bis zur Mündung des Warmatsgundbachs gefunden, ScHulze dieselben von Spielmannsau (14) und RothPletz (13) die gleichen mehrfach zwischen Oberstdorf und Hindelang (inkl. Liebenstein) beschrieben; wenn sie auch meistens an die Basis der ostalpinen Decke geknüpft erșcheinen, so können sie doch kaum alle als zufällig heraufgeholte Schubfetzen gelten, sie dürften weit eher abgepresste Reste des natürlichen, südlichen Flyschbordes vorstellen.

Bezüglich der eigenartigen basischen Eruptiva, die in Zusammenhang mit den Seewerfetzen wie mit den bekannten kristallinen Resten von Oberstdorf und Hindelang (RoTHPLErz 13) gleichfalls scheinbar an die Basis der ostalpinen Decke gebannt sind (lepontinische Späne im Sinne Sternmans's und anderer Autoren), so konnten auch die neueren Untersuchungen PosTopPIDAN's (9) mangels irgend genügender Aufschlüsse keine neuen Beweismittel in einem oder anderem Sinne liefern; zunächst muss somit immer noch das REISER'sche Schürfresultat ${ }^{\mathbf{1}}$ ) (normaler Eruptivkontakt mit Flysch) als unwiderlegt gelten.

Der Schubrand der ostalpinen Masse = Allgäuer Lin ie von RothPLeTz) ist nun von der Künzelspitz bis zum Oytal in etwa $30 \mathrm{~km}$ Länge zusammenhängend und genauestens untersucht worden, so dass sich wohl mit Ergänzung durch Angaben von RoTHPLETz für den noch ausstehenden Streckenverlauf all g e m e in gült i g e Bauregeln ableiten lassen. Als solche dürften zunächst gelten: die Auflagerungsebene ist flach, kaum irgend über $30^{\circ}$, gewöhnlich 10 bis $15^{\circ}$ südöstlich geneigt und nur ausnahmsweise etwas stärker gewellt; die Schichtserie der Decke beginnt über der Schubfläche fast stets mit Hauptdolomit, der kräftig zu Sättel und Mulden verbogen ist; diese Falten der Decken streichen ebenso wie ihre inneren Schuppenflächen frei gegen die Flyschzone aus, deren Falten weder in Art noch Richtung mit jener der Decke übereinstimmen; dagegen durchpflügen verschiedene aus der ostalpinen Masse austretende Querstörungen auch den Schubrand samt Unterlage und setzen mehrfach deutlich in die Flyschzone fort. Öters greift in tiefen, bis $3 \mathrm{~km}$ langen Buchten (Marul, Bregenzer-Ach, Warmatsgundbach, Spielmannsau) das basale Gebirg in die erosionszernagte Decke ein, an verschiedenen Stellen hinwieder lagern einzelne abgeschnürte Deckenteile wie vorgeschobene Inseln vor dem eigentlichen Rand (Osthang des Fellhorns).

Eine der eigenartigsten Erscheinungen bilden die plötzlich auftretenden und rasch wieder verschwindenden, geringmächtigen $\mathrm{K}$ eil e von Aptychenk a lken an der Basis des Deckenrands, die von der Bärenweide, Zwölferspitz, Schüsser, Himmelschrofen $(8,9,14)$ geschildert wurden und welche RoTHPLETz (13) zwischen Oberstdorf

1) Über die Eruptivgesteine des Allgäu. Tscheraak's min. petr. Mitt. X. 1889 
und Hindelang eingehend beschrieben hat; nur äussert selten werden sie von Fleckenmergeln begleitet, dagegen scheinen sie aufs Innigste mit dem Flysch verquält, ja zwischen Flyṣch eingelagert. Sie gleichen durchaus dem einsamen Klippenvorposten des Feuerstättekopfs und müssen deshalb auch wohl gleicher Entstehung sein. Beim langsamen Vorrücken der ostalpinen Decke sind sie wohl bei gelegentlich vorkommender randlicher Uberstülpung von der Oberfläche abgeglitten (1), dainn überfahren und in den Untergrund eingewalzt worden, keinesfalls aber können sie etwa als Relikte des liegenden Schenkels eines gewaltigen Stirngewölbes gedeutet werden; nirgends ist ja in der Hauptdolomitmasse der Kern eines solchen liegenden Sattels erschlossen ja im Gegenteil gerade da, wo derartige Keile erhalten sind, birgt die Decke gar oft stehende Mulden und Sättel (Hölltobel, Elferkopf, Bärenweide), ohne dass die flache Schubbahn im liegenden versehrt wäre.

Für den Innenbau der ostalpinen Decke ist die Kombination kräftiger Falten mit energischer Schuppung charakteristisch. Als wichtigstes Ergebnis der jüngsten Aufnahmen sei die Tatsache vorangestellt, dass diese interne Schuppung (die durch Rothpletz ausgezeichnete ,Lechtaler" Linie mit einbegriffen) unzweifelhaft aus der Überanstrengung einzelner Faltenwellen hervorgegangen ist. Als beste Beispiele hierfür sei zunächst auf die sich in die breite Liasmulde des Rappensees und Heubaumes einkeilende Hebungswele des Grüners und Rauhgernrückens hingewiesen, die Mruus bis gegen den Körbersee verfolgt hat. Während im westlichen Teil (8, Profil 1-4) nur geringe Störungen den normalerweise aus Hauptdolomit, Kössener, roten Lias und Fleckenmergel erbauten stehenden Sattel durchziehen, kommen vom Haldenwanger Eck bei kräftiger Überstürzung zunächst Plattenkalk zu rotem. Lias, dann Hauptdolomit zu Fleckenmergel in einen so auffälligen Schubkontakt, dass RoTHPLE1z hier sogar die grosse Lechtaler Schubmasse vermutet hatte.

Des weiteren legt gerade letztere selbst nach den prächtigen Hanier'schen Profilen das beste Zeugnis ab. Uber dem Hinterhornbachtal stösst sie mit ganz flach verbogener Bahn (Profil I), durch Deckinseln gekennzeichnet, etwa $10 \mathrm{~km}$ weit nach N N W vor (unterster Hauptdolomit auf Fleckenmergeln); mit zunehmender und hier zweifellos ursprünglicher Versteilung gegen West nimmt der stratigraphische Hiatus bis zum Biberkopf Westgrat (im Profil VI Hauptdolomit auf Kössener) zu; gleich darauf taucht (Profil VII) im überschiebenden Teil auch der liegende Schenkel der jetzt engen Sattelw ölbung auf und innerhalb der rhätischen Gesteine des Teschenberggewölbes verbirgt sich die Störung so unauffällig, dass sie Mrıus hier nicht einmal mehr eintragen zu müssen glaubte.

Es ist nun von grossem Interesse, dass durch die MrLius'sehen und Haniez'schen Aufnahmen die Auslösung des Kompensations - 
bedürf nisses solch starken Schuppengefälles aufs Klarste aufgezeigt wird. Während SchuLze für den die Lechtaler Schubmasse unterlagernden ostalpinen Deckenteil im Profil WildengundkopfBurgstall nur eine Schuppe kennt (Himmelschrofen), weisen Mylius' Karte und Profil in Linie Bärenweide-Nordseite des Aarhorns schon 4 sekundäre Schuppenflächen auf. HANIEL beobachtete andererseits innerhalb der Lechtaler Masse an der Ellenbogener Schuppe, wie sie im Osten (am Gibler im Profil 2) lediglich als nahezu saigere Längsverwerfung ein und derselben Mulde von Tithon-Neokom zum Ausdruck kommt, während sie wenige Kilometer westlicher, südlich des: Biberkopfs (Profil 5) schon Hauptdolomit auf Fleckenmergel bringt, ja vielleicht die ganze vorgelagerte Ramstallschuppe bedeckt.

Auch diese sekundären Gleitbahnen zeigen selbst da, wo sie vollständig verflachen, von gelegentlichen, wohl dem unregelmässigen Untergrund sich anschmiegenden Verbiegungen abgesehen, nirgends deutliche Verfaltung, gerade so wie dies für die Auflagerungsfläche der ostalpinen Masse selbst schon festgestellt wurde.

Als schönes Beispiel für die freie Endigung einzelner Faltenwellen am äusseren Schubrand, der unbedingt als echter Erosionsrand, keineswegs als durch interne Bauregeln der ostalpinen Deckevorherbestimmt $\mathrm{zu}$ gelten hat (stets auch deutliche Streichdifferenz zwischen Rand und Innenfalten), sei auf die Mulde des Himmelschrofens hingewiesen, die über Hammerspitz-Schüsser zur Zwölferspitz sich verfolgen lässt und an der Bärenweide als Schuppe $\alpha$ von MyLıus gegen den Deckenrand ausstreicht.

Über die Druckrichtung während des Schuppenstaus kann hente kaum mehr ein Zweifel bestehen; sie muss sich der ganzen. Gestaltung nach ebenso aus südlichen wie östlichen Kompo. nenten zusammensetzen, deren erstere wohl unmittelbar aus dem Verlauf der Falten und Schuppenränder zu entnehmen ist, während für letztere ebenso der ungemein kräftige Vorstoss der Lechtaler Masse östlich des Biberkopfs wie verschiedene Detailbeobachtungen: (z. B. Mylius am Widderstein, Haniel am Nordhang des Muttekopfs). beweisend sind. Gleiche Bewegung ist für die Gesamtbewegung der ostalpinen Decke (nach Rotuplitz ost-westlich, nach Steinmann süd-nördlich) zwar nicht unwahrscheinlich, aber keineswegs sichergestellt; eine Beweisführung allein aus den Verhältnissen des engeren Gebietes heraus konnte ja von vorneherein kaum erwartet werden.

Die Durchforschung der hinteren Lechtaler Alpen, die wir von AMPFERER erwarten dürfen, wird viele einzelne und wertvolle Ergänzungen, doch vermutlich keine wesentlichen Änderungen dieses tektonischen Bildes mit sich bringen. Als zunächstliegendeAufgabe erscheint die Verfolgung der bedeutungsvollen, von AMPFERlik bereits über Boden und Gramais gegen das Griesbachtal festgelegten Heiterwandlinie (4), welche das Vordringen der mächtigen. Klötze des Wettersteins und der Mieminger gegen Nordwest fort-- 
setzt und deren Kompensation, wenn nicht unmittelbare Weiterführung in jener mächtigen bis auf den Muschelkalk hinabgreifenden Schubmasse der Mohnentluh und Braunarlspitz vorliegt, welche Mrcius mit vollem Recht ganz besonders hervorgehoben, wenn auch irrtümlicherweise als "Lechtaler"schuppe bezeichnet hat.

Es exübrigt noch die Hauptzüge der Entstehungsgeschichte dieses Teiles der Nordalpen vor allem auch in zeitlicher Beziehung miteinander in Verbindung zu bringen.

Nach Ablagerung des Neokoms setzen innerhalb der os tal pin en Masse zuerst wieder tektonische Schwankungen grösseren Ausmasses ein in scharfem Gegensatz zur helvetischen Zone, in welcher zwischen Schrattenkalk und Gault nur ganz geringfügige Sedimentationsdiskordanz nachweisbar ist. Erst das zenomane und später das senone Meer dringen mit energischer Abrasion, im Muttekopfgebiet bis auf den Hauptdolomit sich einfressend, von Nord gegen Süd vorschreitend, in das ostalpine Deckengebiet wieder ein. Im Senon machen sich allerdings auch in der helvetischen Zone rascher erfolgte Strömungs- und Niveauveränderungen in dem reicheren, oft an ostbayerische Verhältnisse anklingenden Wechsel der Fazies bemerkbar, erst mit der Wende zum Tertiär entsteht dann aber gleichfalls ein harter Sedimentationshiatus, den das basale Grundkonglomerat des un tereozänen Flys ch es beleuchtet.

Nach ToRNQUrst im älteren, nach RothPLETz zwischen unterem und oberem Oligozän ist das Vor rücken der ostalpinen Deckenmasse anzusetzen; diese ist in jenem Augenblick bereits in kräftige Faltenreihen gelegt, die von der Schubbahn schräg geschnitten werden, ihrerseits wieder an zahlreichen Stellen aufrissen und so sekundäre Schuppenbildung veranlassten, nach der RothrLeTz'schen Auffassung gelegentlich des Vordringens der ostalpinen Masse selbst infolge Untergrundstauchungen und Verkeilungserscheinungen innerhalb derselben. Der grössere Teil der auftretenden Verwerfungen dürfte wohl gleichen Alters sein, da sie ja öfters deutlich sich auf eine Schuppe beschränkt zeigen (7).

Ausserhalb des deckenbeschwerten Landes erfolgte gleichzeitig die Ablagerung des jüngeren Flysehes, ausgezeichnet durch Einverleibung vieler fremder, von den Decken herstammender Gerölle (ToRnquist).

Im Oberoligozän ist die grosse ostalpine Massenverfrachtung zum Stillstand gekommen, auch die Klippen des Feuerstätterkopfs sind dem Flyschuntergrund bereits aufgeladen.

In das ältere Miozän möchte ToRNQurst, allerdings ohne dafür in jeder Beziehung stichhaltige Gründe angeben zu können, die Auffaltung der Kreideketten und der Flyschzone sowie die Aufpressung der helvetischen Kreide auf den nördlichen Flyschstreif ansetzen. Die starke Aberosion des ostalpinen Deckenlands in der Linie Oberstdorf-Riezlern wäre somit wesentlich durch diese tektonischen Er- 
eignisse begünstigt. Auch eine nicht unbeträchtliche Anzahl von Störungen (z. B. Trettachlinie, Hochstättenbruch), die den ostalpinen Schubrand nebst Untergrund ebenso wie die Flyschkreidegrenze durchsetzen, aber an der Molasselinie ihr Ende finden $(15,16)$, könnte dieser Faltungsperiode zuzurechnen sein.

In das obere Miozän endlich dürfte die Wellung der Molasse sowie der Anschub des Flysches auf diese zu verlegen sein.

So sehr dieses Schema auch der Nachprüfung späterer Untersuchungen bedürftig sein mag, das eine scheint festzustehen, dass seit Ende der Neokomzeit sich sammelnde, bis ins Oligozän sich verstärkende und'erst'imjüngsten Tertiär sich verlierende Druckkräte in diesem Teile der Alpen grossartige Krustenbewegungen vorwiegend tangentialen Charakters auswirkten, wobei sich langsam der Höhepunktdes gebirgsbildenden Aufstaus vom Inneren deralpinen Massengegendas Vorland verschob.

\title{
Nenere Arbeiten über die regionale Geologie des Mainzer Beckens.
}

\author{
Von C. Mordziol (Aachen).
}

Mit Fig. 1.

Seit der Zusammenfassung in LePsius' Geologie von Deutschland sind zahlreiche Spezialarbeiten über das Mainzer Becken erschienen, die eine neue, kurze Zusammenstellung notwendig machen. Ihnen allen in einem einzigen Sammelreferat auch nur annähernd gerecht zu werden, ist kaum möglich. Es werden daher an dieser Stelle zunächst nur die Arbeiten berücksichtigt, die für Fragen von mehr allgemeiner Natur von Bedeutung sind. Solche Fragen beziehen sich vornehmlich auf das Verhältnis von Meeressand zu Septarienton, die Gliederung des Septarientons, Gliederung und Alter der sog. Cyrenenmergelgruppe und der oberen Abteilung des Mainzer Tertiärs, Entstehung und Alters-. stellung des Eppelsheimer Sandes, Gliederung und Parallelisierung der Diluvialterrassen und des Löss. Manche, an und für sich sehr wichtige stratigraphische Arbeiten mussten einstweilen zurückgestellt werden.

Noch einige zusammenfassende neuere Darstellungen seien erwähnt, so vor allem Kinkelin's Abhandlung über „Die Tertiärund Diluvialbildungen des Untermaintales, der Wetterau usw. in den Abhandlungen zur geol. Spezialkarte von Preussen. Bd. IX. Heft 4. Berlin 1892. Ferner findet man eine solche in Nr. 3 des nun folgenden Literaturverzeichnisses, in "Offenbach in Wort und Bild" (ron K. VöLzing, mit besonderer Berücksichtigung der Entwickelung der 